\title{
FABRICATION OF MAGNETIC MICROSTRUCTURES BY IN SITU CROSSLINKING OF MAGNETICALLY ASSEMBLED NANOPARTICLES
}

\author{
Camilo Velez ${ }^{{ }^{*}}$, Isaac Torres-Díaz ${ }^{2}$, Lorena Maldonado-Camargo ${ }^{3}$, Carlos Rinaldi ${ }^{2,3}$ and David P. Arnold ${ }^{1}$ \\ ${ }^{1}$ Department of Electrical \& Computer Engineering, University of Florida, USA \\ ${ }^{2}$ J. Crayton Pruitt Family Department of Biomedical Engineering, University of Florida, USA \\ ${ }^{3}$ Department of Chemical Engineering, University of Florida, USA
}

\begin{abstract}
We present a photolithography-free fabrication technique to create magnetic microstructures using magnetically self-assembled iron oxide $\left(\mathrm{Fe}_{3} \mathrm{O}_{4}\right)$ nanoparticles, which are subsequently crosslinked to each other via surface polymerization. This effort is a step toward low-cost roll-to-roll fabrication of free-floating micromagnetic actuators such as microswimmers, micro-stirrers, micro-tweezers, etc. Four main accomplishments are reported: (1) optimization of the particle deposition (self-assembly) conditions to achieve feature sizes down to $5 \mu \mathrm{m}$, (2) development of the polymerization process to crosslink the nanoparticles, (3) release of the microstructures from the substrate via a sacrificial layer, and (4) demonstration of the magnetic actuation of prototype structures.
\end{abstract}

\section{INTRODUCTION}

Magnetic microstructures are of growing interest for fluidic, biomedical, and even optical applications. A conventional "MEMS fabrication" approach for achieving such structures involves lithography, thin-film magnetic material deposition, and liftoff or etching [1], but such processes require complex, expensive fabrication tools. An alternative way to create magnetically responsive microstructures is by embedding magnetic particles in photoactive polymers [2]. However, the particle loading fraction is typically low to maintain photolithographic patternability. We introduce here a new method that uses magnetically directed selfassembly (rather than photolithography) to form microstructures of various sizes and shapes. Another group has used this concept to pattern magnetic nanoparticles on hard disk drive substrates to create optical gratings [3]. However, we extend this idea by crosslinking the nanoparticles using polymers and then releasing them from the substrate, with the aim of forming functional magnetic micro-components. Some groups have worked on crosslinking of magnetic nanoparticles for applications such as construction of biological scaffold materials for tissue engineering [4], fabrication of bio hydrogels for controlled drug delivery [5],[6], modification of optical properties in the creation of diffraction gratings [7], and separation of food dyes from water [8]. However, in all these cases the particles are crosslinked in to spheres or loose aggregates and do not possess more complex structure. Our approach facilitates flexible fabrication of complex microstructures that are mostly magnetic, rather than polymer, for maximum magnetic responsiveness.

\section{EXPERIMENTAL METHODS}

The overall process is shown in Fig. 1. First, superparamagnetic iron oxide $\left(\mathrm{Fe}_{3} \mathrm{O}_{4}\right)$ nanoparticles (in this case, $17 \mathrm{~nm}$ diameter) are synthesized by thermal decomposition of an iron oleate precursor. The resulting oleic acid coating is oxidized to form carboxylic acid, which confers colloidal stability and permits subsequent crosslinking using polyamines. The nanoparticles are suspended in aqueous solution and then magnetically self-assembled onto a substrate, wherein regions of high field-gradient define the assembly pattern (e.g. line, circle, etc.). The field-gradient regions are created by spatially magnetized patterns in a magnetic tape, previously coated with a sacrificial layer. After the particles are assembled, they are crosslinked using branched polyethylenimine (PEI). The microstructures formed by this process are then released from the substrate by dissolving the sacrificial layer. More details on each of these steps are provided in subsequent sections.

\section{Magnetic Nanoparticles}

Iron oxide nanoparticles were synthesized by thermal decomposition of an iron oleate precursor using procedures described in [9]. This method yields magnetic particles coated with a monolayer of oleic acid, making them soluble only in organic solvents. However, the crosslinking method used in our experiments must be done in a water solution. Hence, the particles were converted to an aqueous solution by using a strong oxidizing agent to cleave the double bond present in the oleic acid chain obtaining carboxylic acid terminal groups [10], as shown in Fig. 2(a). We obtained negatively charged and electrostatic stabilized particles for crosslinking to polyamines.

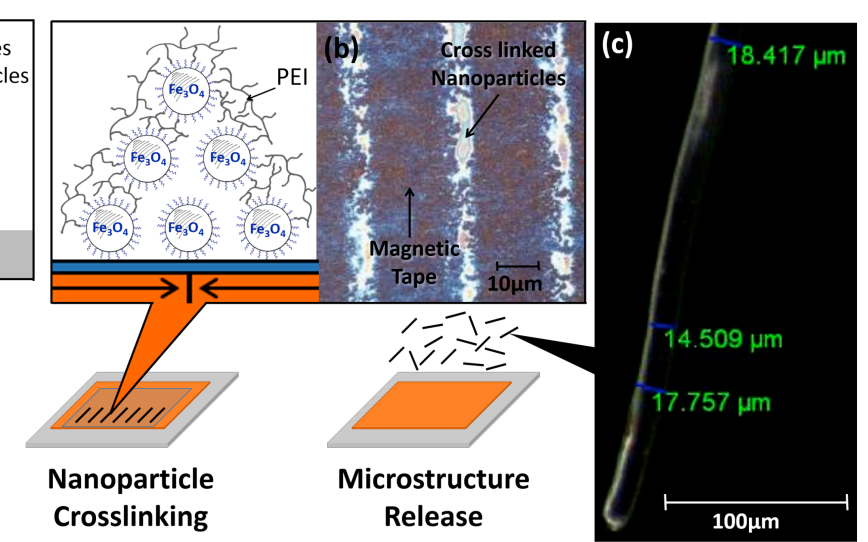

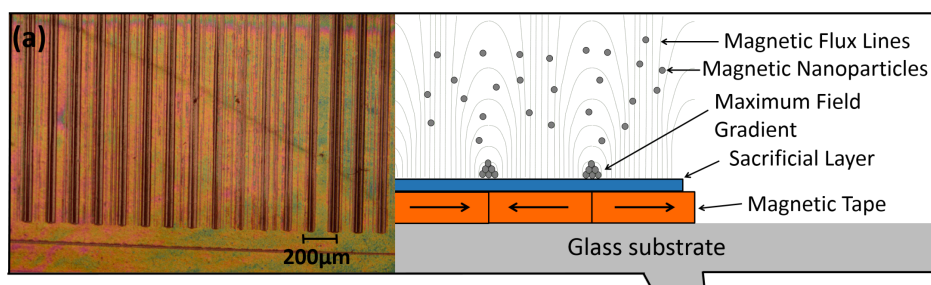

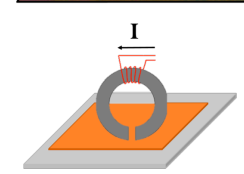

Magnetic Patterning
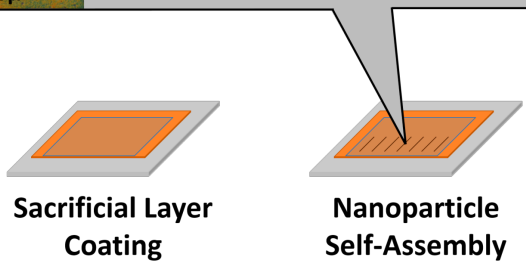

Figure 1: Fabrication method: (a) Nanoparticles self-assembled onto magnetically patterned substrate (audio cassette tape). (b) Nanoparticle surface modification and cross-linking with PEI using EDC and NHS. (c) Sacrificial layer dissolved, releasing magnetic structures. 
The particles were characterized using dynamic light scattering (DLS) to measure the hydrodynamic diameter $(28.0 \pm$ $1.18 \mathrm{~nm})$. The physical diameter $(17.0 \pm 0.1 \mathrm{~nm})$ was characterized by transmission electron microscopy (TEM) as shown in Fig. 2(c). Magnetic properties of the particles were also measured using a superconducting quantum interference device (SQUID) magnetometer. The data was fit to the Langevin equation using the procedure suggested by Chantrell [11] to estimate the magnetic particle size distribution of the sample, shown in Fig. 2(b). From this, the magnetic volume fraction $\varphi$ was $0.062 \% \mathrm{v} / \mathrm{v}$, and the magnetic core diameter $\mathrm{D}_{\mathrm{p}}=9 \mathrm{~nm}$ and $\ln (\sigma)=$ 0.44 . These results confirm monodispersity of the nanoparticles used during the experiments.
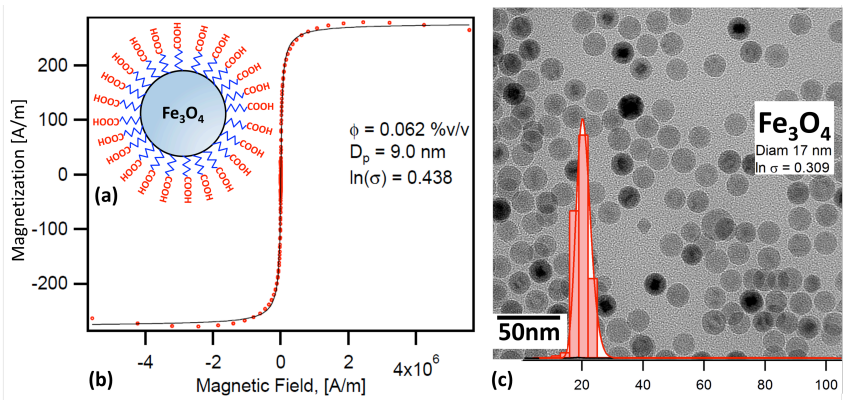

Figure 2: $\mathrm{Fe}_{3} \mathrm{O}_{4}$ magnetic nanoparticles. (a) Nanoparticle schematic showing the $\mathrm{COOH}$ group, (b) Magnetic hysteresis curve measured using SQUID. Red dots represent measured data, while black line represents the fitting of Langevin equation to the curve, (c) Background: TEM image of the nanoparticles. Foreground: size distribution of the particles via TEM image.

\section{Magnetic Patterning of Cassette Tape Substrates}

An audiocassette tape was used as a magnetic substrate to magnetically assemble the particles into pre-defined patterns, inspired by [12]. In initial experiments, the tape was recorded with a sinusoidal wave of $500 \mathrm{~Hz}$ at $20 \mathrm{kHz}$ sampling rate to generate samples with simple line patterns with a fixed period of $25 \mu \mathrm{m}$. These lines were used to characterize the process and to generate a better understanding of the variables involved in assembly. In later experiments, samples of tape were selectively magnetized to generate arbitrary patterns using procedures reported in [13],[14]. Several example patterns are shown in Fig. 3.
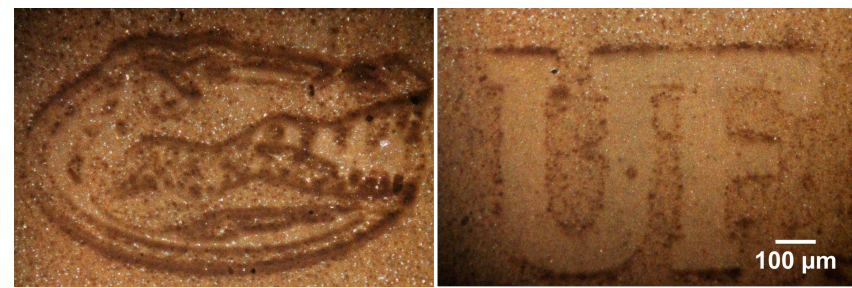

Figure 3: Magnetic nanoparticles assembled in arbitrary patterns. Deposition time: 20 hours.

Before self-assembly of the particles, the magnetically patterned tape was coated with a sacrificial layer. Two different sacrificial layers were evaluated: (1) $350 \mathrm{~nm}$ of LOR 3B (polydimethylglutarimide based resist) and (2) $85 \mathrm{~nm}$ of PMMAA2 (polymethyl methacrylate). The sacrificial layer has an important role in the particle deposition and must be chosen carefully. The conditions of the nanoparticles and the sacrificial polymer can generate high hydrophobicity, thereby inhibiting deposition or weakening dramatically the adhesion of the nanoparticles to the magnetized substrate. Another important aspect of the sacrificial layer is the thickness. Thick sacrificial layers can enhance the contrast between areas with particles and those without particles, but if the layer is too thick the particles may not assemble at all.

\section{Self-Assembly of Magnetic Particles}

The magnetic particle self-assembly was made by immersing the magnetized tape in the nanoparticle suspension. Particles deposited in regions of high field-gradient on the magnetized tape generating defined assembly patterns (e.g. line, circle, letters, etc.), as shown in Fig. 3. A gentle rinse, using $0.5 \mathrm{~mL}$ of water with $\mathrm{pH}$ 11 (same $\mathrm{pH}$ of the nanoparticles suspension) and surface inclination was necessary to eliminate excess particles surplus from the sample and to finalize the deposition.

The experiments were conducted by parametrically varying various self-assembly parameters (time, particle concentration, thickness of sacrificial layer, orientation of the magnetic tape during the deposition, and washing conditions) to determine their influence on the line width of the deposited structures.

The most significant parameter was found to be the nanoparticle concentration, which showed positive correlation with the line width. Figure 4 shows how the line width of the assembled particles could be controlled from $14 \mu \mathrm{m}$ down to $\sim 5$ $\mu \mathrm{m}$ by controlling the particle concentration from $0.36 \%$ to $0.0021 \%$ by volume for a 5 -min deposition time.

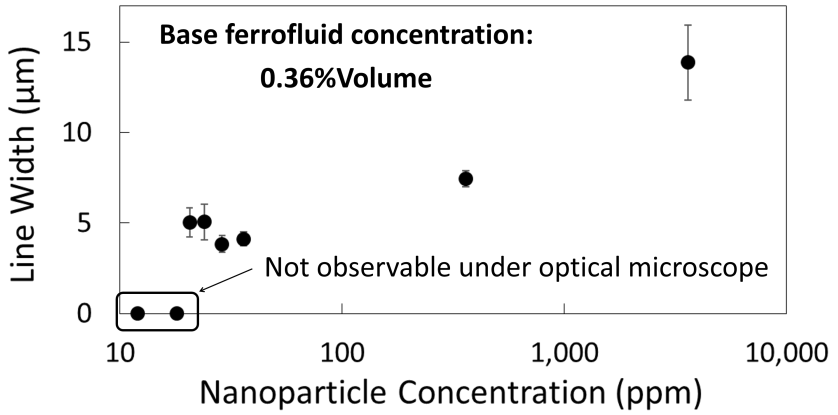

Figure 4: Relationship between concentrations of nanoparticles in suspension and the linewidth of the magnetic structure for 5 minutes of deposition.

Another critical parameter was found to be the deposition time. When the particle concentration is constant, there was a positive correlation of the line width with deposition time. Figure 5 shows results from a qualitative analysis of the effect of deposition time using values varying logarithmically from 6 seconds to 600,000 seconds. For this experiment, the particle concentration was low $(0.062 \% \mathrm{v} / \mathrm{v})$, requiring very long deposition times in order to obtain well-defined patterns.

\section{Particle Crosslinking and Microstructure Release}

After particle assembly, a $25 \%$ weight solution of the branched poly-cation polyethylenimine (PEI) with molecular weight of $2 \mathrm{kDa}$ was used to crosslink the carboxylic acid groups present on the nanoparticle surfaces with the primary amines in the polymer chain. This reaction was carried out for 1 hour and promoted by 1-ethyl-3-[3-dimethylaminopropyl] carbodiimide hydrochloride (EDC) and N-hydroxysuccinimide (NHS) chemistry [15-18]. A final rinse with deionized water and acetic acid was used to remove free polymer.

To illustrate the results of successful crosslinking, Fig. 6 presents SEM images of the simple line patterns from the audiocassette recording. The light colored regions indicate the 
presence of polymer (electrically insulated), meanwhile dark regions represent the magnetic tape (electrically conductive). Figure 6(b) shows an enlarged image of the end of one of the lines to show the agglomeration of crosslinked nanoparticles.

Figure 7 shows images of a more complex, patterned grid microstructure. Figure 7(a) shows the magnetization mask used for the selective magnetization process, and Fig. 7(b) shows the selfassembled and crosslinked patterns before release. Figure 7(c) shows a corresponding SEM image of the patterned grid microstructure after crosslinking.
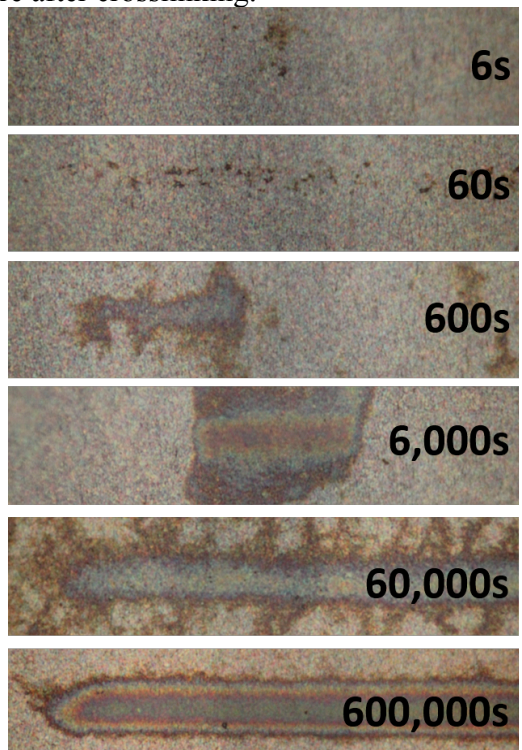

Figure 5: Time deposition qualitative analysis. Particle volume fraction in suspension $\varphi=0.062 \% \mathrm{v} / \mathrm{v}$.

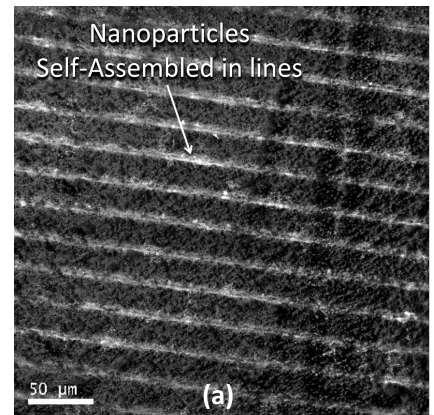

Figure 6: SEM top view nanoparticles on magnetic tape, (b) bonded particle microstructure after crosslinking.

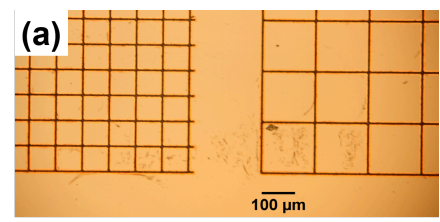

(b)
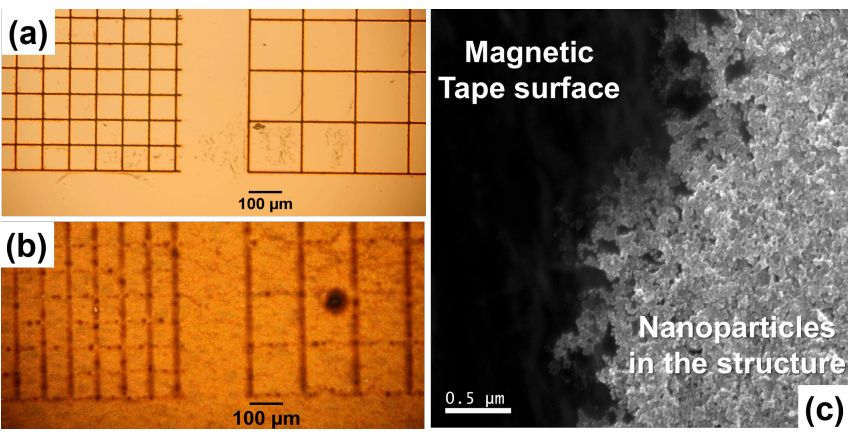

Figure 7: Grid microstructure: (a) Magnetization mask used to create the pattern, (b) crosslinked self-assembled nanoparticles and (c) SEM top view picture of the structure boundary.
Lastly, the underlying sacrificial layer (350 nm of LOR 3B) was dissolved in AZ300 MIF developer (tetra-methyl ammonium hydroxide), releasing the structures into solution. Figure 8 shows an optical image of the released grid structure and distortion of the grid pattern when subjected to a magnetic field. These results prove the successful crosslinking of the magnetic nanoparticles into the patterned microstructures and the magnetic response of said complex structure.

After releasing the sacrificial layer smaller microstructures will float in the releasing solvent, and these free-floating microstructures were shown to be highly responsive to external magnetic fields. Experiments with random variations of surrounding magnetic field applied to free-floating structures resulted in the ability to rotate the structures about different axes, as in Fig. 9. Figure 10 shows flexing of a structure that was partially attached to the substrate. When the changing magnetic field was applied part of the structure bent towards the magnetic field source. When a field was inverted the bending was in the opposite direction. These experiments illustrate the potential application of this fabrication technique to obtain structures that can be actuated magnetically.
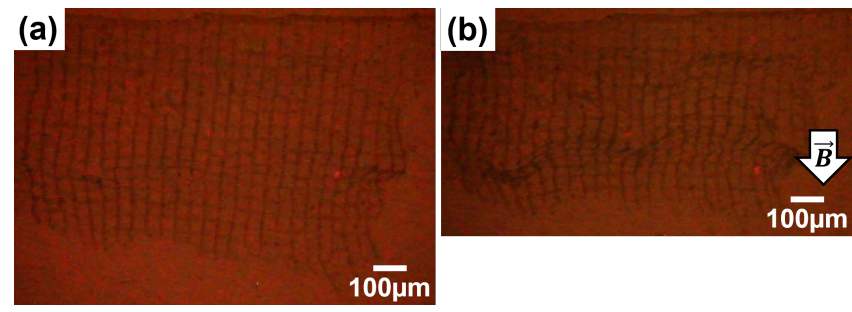

Figure 8: Released grid microstructure: (a) released structures in suspension (b) released structures responding to the presence of a magnetic field, showing distortion of the grid.
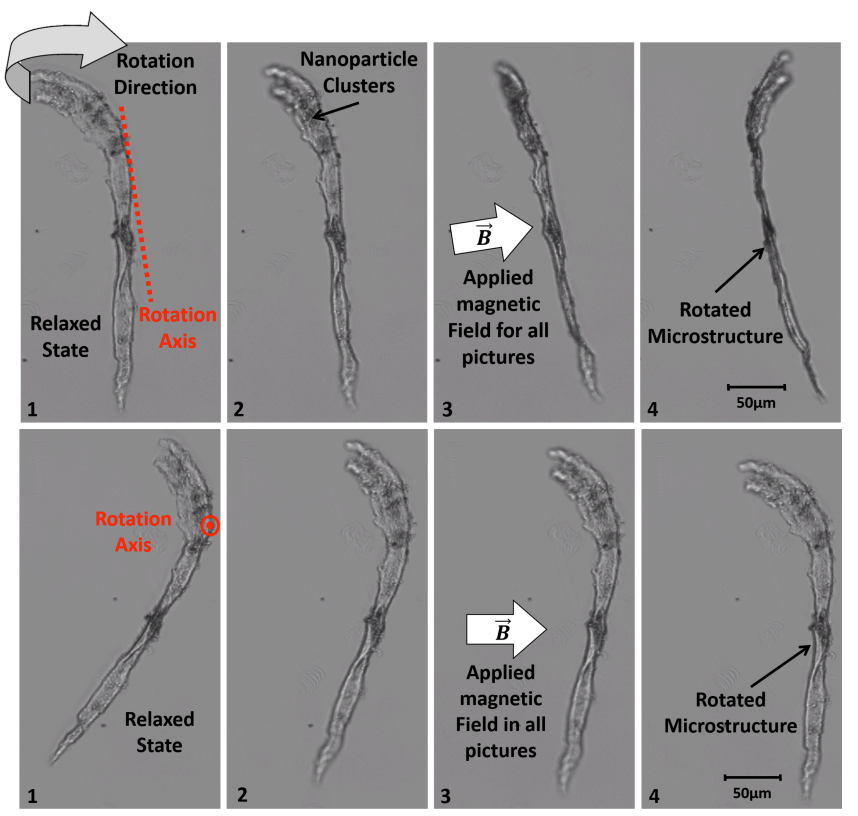

Figure 9: Rotation experiments on microstructure changing the orientation of the magnetic field. 


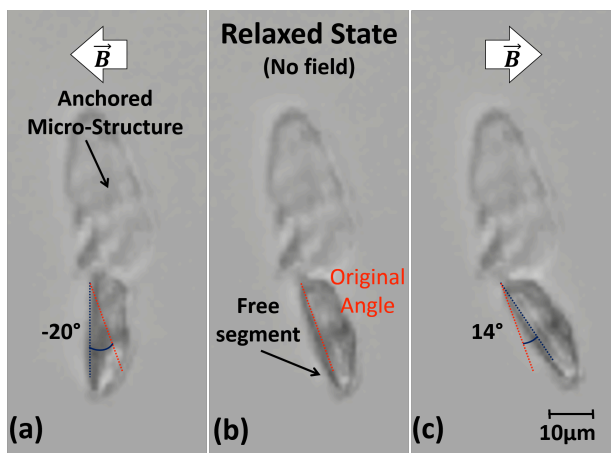

Figure 10: Response of microstructure to the presence of external magnetic field. Panel (b) shows the relaxed state of the structure, whereas panels (a) and (c) represent position after excitation to the left and right, respectively.

\section{CONCLUSIONS}

A technique for fabrication of magnetic microstructures by in situ crosslinking of magnetically assembled nanoparticles was demonstrated. Two strategies to create magnetic field gradients to pattern the nanoparticles were evaluated: Recording media data (producing rectangles with linewidth around $10 \mu \mathrm{m}$ and length of 1 $\mathrm{mm}$ ) and a selective magnetization technique (to produce arbitrary patterns). Both strategies were successful and generated microstructures that were successfully released in solution. SEM pictures demonstrate the high density of particles inside the structures, and actuation by external magnetic fields validates their magnetic responsiveness.

The most critical parameters for the process are: nanoparticle concentration, deposition time, magnetic field during the selective magnetization, sacrificial layer chemistry and thickness and microstructure release. Further experimentation must be done in order to optimize these parameters for specific structure shapes. The releasing process is a critical step that must be optimized in the future for better conservation of the free microstructures. These results prove the concept of the process for creating more complex shapes, patterns, and functional devices in the future.

\section{ACKNOWLEDGEMENT}

This work is funded in part by the UF Office of Research. The authors thank Ololade Oniku and Dr. Alexandra Garraud for their contribution as well as the staff of the UF Nanoscale Research Facility and the UF Major Analytical Instrumentation Center for their assistance in the microfabrication and material characterization.

\section{REFERENCES}

[1] B. Dieny, H. Joisten, and P. Sabon, "Magnetic microparticle and method for making such a microparticle," International Patent WO201103308025-Mar-2011.

[2] J. Kim, S. E. Chung, S.-E. Choi, H. Lee, J. Kim, and S. Kwon, "Programming magnetic anisotropy in polymeric microactuators.," Nat. Mater., vol. 10, no. 10, pp. 747-52, Oct. 2011.

[3] J. Henderson, S. Shi, S. Cakmaktepe, and T. M. Crawford, "Pattern transfer nanomanufacturing using magnetic recording for programmed nanoparticle assembly.," Nanotechnology, vol. 23, no. 18, p. 185304, May 2012.

[4] E. Alsberg, E. Feinstein, M. P. Joy, M. Prentiss, and D. E. Ingber, "Magnetically-Guided Self-Assembly of Fibrin Matrices with," Tissue Eng., vol. 12, no. 11, pp. 3247-3256, 2006.
[5] R. Barbucci, G. Giani, S. Fedi, S. Bottari, and M. Casolaro, "Biohydrogels with magnetic nanoparticles as crosslinker: characteristics and potential use for controlled antitumor drug-delivery.," Acta Biomater., vol. 8, no. 12, pp. 4244-52, Dec. 2012.

[6] S. Sun, S. Anders, H. F. Hamann, J.-U. Thiele, J. E. E. Baglin, T. Thomson, E. E. Fullerton, C. B. Murray, and B. D. Terris, "Polymer mediated self-assembly of magnetic nanoparticles.," J. Am. Chem. Soc., vol. 124, no. 12, pp. 2884-5, Mar. 2002.

[7] L. Ye, B. Terry, O. T. Mefford, C. Rinaldi, and T. M. Crawford, "All-nanoparticle concave diffraction grating fabricated by self-assembly onto magnetically-recorded templates.," Opt. Express, vol. 21, no. 1, pp. 1066-75, Jan. 2013.

[8] Z. Zhou, S. Lin, T. Yue, and T.-C. Lee, "Adsorption of food dyes from aqueous solution by glutaraldehyde cross-linked magnetic chitosan nanoparticles," J. Food Eng., vol. 126, pp. 133-141, Apr. 2014.

[9] J. Park, K. An, Y. Hwang, J.-G. Park, H.-J. Noh, J.-Y. Kim, J.-H. Park, N.-M. Hwang, and T. Hyeon, "Ultra-large-scale syntheses of monodisperse nanocrystals.," Nat. Mater., vol. 3, no. 12 , pp. 891-5, Dec. 2004.

[10] M. Wang, M.-L. Peng, W. Cheng, Y.-L. Cui, and C. Chen, “A novel approach for transfering oleic acid capped Iron Oxide Nanoparticles to water phase," J. Nanosci. Nanotechnol., vol. 11, no. 4, pp. 3688-3691, 2011.

[11] R. Chantrell, J. Popplewell, and S. Charles, "Measurements of particle size distribution parameters in ferrofluids," IEEE Trans. Magn., vol. 14, no. 5, pp. 975-977, Sep. 1978.

[12] A. J. Moreno, E. Gonzalez, M. Godoy, J. Pettinari, P. S. Antonel, G. Jorge, and V. Bekeris, "Spatial Resolution in Micrometric Periodic Assemblies of Magnetotactic Bacteria and Magnetic Nanoparticles," IEEE Trans. Magn., vol. 49, no. 8, pp. 4572-4575, Aug. 2013.

[13] A. Garraud, O. D. Oniku, W. C. Patterson, E. Shorman, D. Le Roy, N. M. Dempsey, and D. P. Arnold, "Microscale magnetic patterning of hard magnetic films using microfabricated magnetizing masks," in MEMS 2014, 2014, pp. 520-523.

[14] O. D. Oniku, P. V. Ryiz, A. Garraud, and D. P. Arnold, "Imprinting of fine-scale magnetic patterns in electroplated hard magnetic films using magnetic foil masks," J. Appl. Phys., vol. 115, no. 17, p. 17A718, May 2014.

[15] A. López-Cruz, C. Barrera, V. L. Calero-DdelC, and C. Rinaldi, "Water dispersible iron oxide nanoparticles coated with covalently linked chitosan," J. Mater. Chem., vol. 19, no. 37, p. 6870, 2009.

[16] X. Wang, L. Zhou, Y. Ma, X. Li, and H. Gu, "Control of aggregate size of polyethyleneimine-coated magnetic nanoparticles for magnetofection," Nano Res., vol. 2, no. 5, pp. 365-372, Mar. 2010.

[17] H. H. P. Yiu, S. C. McBain, Z. a D. Lethbridge, M. R. Lees, and J. Dobson, "Preparation and characterization of polyethylenimine-coated Fe3O4-MCM-48 nanocomposite particles as a novel agent for magnet-assisted transfection.," $J$. Biomed. Mater. Res. A, vol. 92, no. 1, pp. 386-92, Jan. 2010.

[18] S. A. Madison and J. O. Carnali, "pH Optimization of Amidation via Carbodiimides," Ind. Eng. Chem. Res., vol. 52, no. 38, pp. 13547-13555, Sep. 2013.

\section{CONTACT}

*Camilo Velez, tel: +1-352-575-7581; camilovelez@ufl.edu 